\title{
BANCO DE SEMENTES DO SOLO DE MARGEM VIÁRIA DOMINADA POR CAPIM-ANNONI-2 E SUJEITO AO CONTROLE COM DISTÚRBIOS NO SOLO E INTRODUÇÃO DE GRAMÍNEAS ${ }^{1}$
}

\author{
NADILSON ROBERTO FERREIRA², RENATO BORGES DE MEDEIROS ${ }^{3}$, RODRIGO FAVRETO ${ }^{4}$
}

\begin{abstract}
RESUMO - Os impactos das ações antrópicas nas rodovias favorecem um ecossistema diferenciado do seu entorno, facilitando o estabelecimento de espécies oportunistas, como o capim-annoni-2 (Eragrostis plana Nees). No Rio Grande do Sul os acostamentos de rodovias encontram-se invadido por esta espécie, constituindo-se como centro dispersor. Com este estudo objetivou-se descrever o tamanho e a composição do banco de sementes do solo (BSS) deste ambiente, e demonstrar o seu potencial para recompor a florística campestre de acostamentos degradados e invadidos através do estimulo do BSS. O experimento foi estabelecido à margem de uma estrada municipal, anexa à sede da Fazenda São Lucas, no município de Rio Pardo RS, no período de janeiro de 2005 a maio de 2006. O delineamento foi em blocos casualizados com parcelas subdivididas e com três repetições. Os tratamentos das parcelas foram: uso de subsolador e de subsolador mais grade com aplicação de calcário e fósforo. Nas subparcelas, foram semeadas Megathyrsus maximus, Setaria sphacelata, mistura de três espécies nativas de Paspalum ssp e exclusão (testemunha). A ocorrência de sementes de espécies exóticas representou $20 \%$ do BSS, destacando-se o capim-annoni-2. As espécies inibidoras do BSS do capim-annoni-2 foram Megathyrsus maximus e Setaria sphacelata, associadas à operação de subsolagem, gradagem e adubação. O tamanho e a riqueza do BSS indicam alto potencial de regeneração da vegetação herbácea nativa em acostamentos de rodovias invadidos por capim-annoni-2.
\end{abstract}

Termos para indexação: impactos, acostamentos, espécies exóticas, Eragrostis plana Nees.

\section{SOIL SEED BANK OF THE ROADSIDE DOMINATED BY ANNONI-2-GRASS AND SUBMITED TO THE CONTROL BY SOIL DISTURBANCE AND GRASS SPECIES INTRUDUCTION}

\begin{abstract}
The impacts of the anthropic actions in roadsides promotes a differenciated ecosystem in its surroundings facilitating the establishment of opportunist species like annoni-2-grass (Eragrostis plana Nees). The roadsides of Rio Grande do Sul state, in southern Brazil, are invaded by this weed specie forming dispersion centers. The objective of this study was to evaluate the soil seed bank (SSB) size and composition in these roadsides, and demonstrate its potential to regenerate the original grassland of these degraded and invaded areas. The trial was established on roadside next to the São Lucas Farm headquarters, in Rio Pardo county RS, from January 2005 to may 2006. The experimental design was a split-plot type in complete blocks, with tree replications. The plots were submitted to two treatments: deep sub-soil tillage and deep sub-soil tillage plus light soil disking
\end{abstract}

\footnotetext{
${ }^{1}$ Submetido em 10/07/2007. Aceito para publicação em 03/02/2008. Parte da Dissertação de Mestrado do primeiro autor no Programa de PósGraduação em Zootecnia - UFRGS;
}

${ }^{2}$ Eng. Agrônomo, MSc., Departamento de Plantas Forrageiras e Agrometeorologia, UFRGS, nr.ferreira@uol.com.br;
${ }^{3}$ Professor Adjunto, Dep. Plantas Forrageiras e Agrometeorologia, Fac. Agronomia, UFRGS, Av. Bento Gonçalves, 7712, CEP: 91540-000, Porto Alegre-RS. medeiror@orion.ufrgs.br;

${ }^{4}$ Eng. Agrônomo, MSc., FEPAGRO Litoral Norte, RS 484 km 05, CEP: 95530-000, Maquiné, RS. rfavreto@fepagro.rs.gov.br. 
plus limestone and phosphate application. The split-plots were sowed with Megathyrsus maximus, Setaria sphacelata, a mixture of seeds of three native Paspalum sp. and exclusion. The percentage of exotic seed species represented $20 \%$ of the SSB composition in which annoni-2-grass presented the highest seed percentage. The inhibitor species of annoni-2-grass SSB were Setaria sphacelata and Megathyrsus maximus associated with the use of deep sub-soil tillage plus light soil disking plus fertilization. The size and richness of the SSB showed high potential to regeneration of native vegetation in roadsides invaded by annoni-2-grass.

Index terms: impacts, roadside, exotic species, Eragrostis plana Nees.

\section{INTRODUÇÃO}

Os severos impactos sofridos nos ecossistemas das margens de rodovias, iniciando-se na construção das estradas, com perda de bioestrutura, fertilidade e aumento da compactação, promovem um ambiente diferenciado do seu entorno (Primavesi, 1979). Estes eventos induzem mudanças na estrutura da comunidade, promovendo o surgimento de espécies invasoras. Neste contexto o banco de sementes do solo (BSS) tem importância na regeneração da cobertura vegetal nativa e é tido como a "memória genética" das espécies ali presentes, mostrando heterogeneidade na composição entre as sementes transitórias e permanentes. (Cavers, 1995; Fenner, 1995; Bekker, 1998).

Nos acostamentos das estradas, a interação da intensidade, freqüência e escala dos distúrbios deixa o sistema em constante disclímax antropogênico (Odum, 1988), permitindo o surgimento de espécies oportunistas, algumas sendo invasoras, como o capim-annoni-2 (Eragrostis plana Nees), com foco dispersor nas margens das estradas. Esta invasora é uma Poaceae de hábito cespitoso, perene, estival, com origem nas savanas africanas (Boldrini e Kampf, 1977). Sua entrada no Rio Grande do sul ocorreu nos anos 50, como contaminante de sementes importadas de Chloris gayana Kunth e Eragrostis curvula Schrader. A semente é pequena, $(0,14 \times 0,06 \times 0,04 \mathrm{~cm})$, de coloração vermelhoalaranjada a castanho (Kissmann, 1991), com peso médio de mil sementes 0,21g (Medeiros et al., 2004), e considerada como componente do BSS persistente permanecendo viável no solo por mais de cinco anos (Medeiros et al.; 2005).

Os impactos são mais intensos nos primeiros três metros da faixa de domínio da rodovia, e observa-se a formação de uma micro-paisagem de mosaico (Pickett e White, 1985), onde vários estágios serais estão presentes, obedecendo a um gradiente crescente de riqueza e complexidade das comunidades bióticas que se inicia da borda do pavimento em direção às cercas (Forman e Heanue, 2002). Muitas espécies desses habitats são generalistas, prostradas, de grande amplitude de tolerância e adaptadas a estes ambientes microclimáticos, onde o BSS reflete a história dos distúrbios no local (Forman e Heanue, 2002).

O BSS dos acostamentos viários possui uma função estratégica de sustentabilidade ambiental dos campos adjacentes, não sendo contabilizada nos cálculos econômicos, daí a necessidade de uma nova visão biofísica com o conceito de economia ecológica (Costanza, 1991).

A prevenção de invasão biológica começa na restauração da vegetação nativa e pode ser acelerada com a introdução de espécies de forrageiras cultivadas adaptadas, que funcionam como barreiras naturais (Forman e Heanue, 2002). O objetivo deste estudo foi conhecer o padrão do BSS dos ecossistemas dos acostamentos viários da Depressão Central, RS, descrever sua composição, tamanho e inferir sobre o seu potencial em regenerar a vegetação nativa destes locais hoje invadidos por capim-annoni-2.

\section{MATERIAL E MÉTODOS}

O experimento foi estabelecido em 07 de abril de 2005 em um acostamento viário municipal, dominado por capimannoni-2, situado próximo da sede da Fazenda São Lucas, km 197 da BR 290, na região eco-climática da Depressão Central, no município de Rio Pardo, RS. De acordo com a classificação de Köeppen o clima da região é caracterizado como tipo $\mathrm{Cfa}$, e solo classificado como argissolo vermelho distrófico latossólico - PVd4 (Streck et al., 2002).

$\mathrm{O}$ delineamento experimental foi implantado em blocos casualizados com parcelas subdivididas e três repetições. As parcelas foram seis e cada uma subdividida em quatro totalizando 24 subparcelas, locadas de forma contínua no 
acostamento. Cada subparcela nas dimensões de $4 \mathrm{~m} \times 6 \mathrm{~m}$, totalizou uma área de $576 \mathrm{~m}^{2}$.

As parcelas foram submetidas a dois tratamentos: subsolagem (S) e subsolagem, gradagem e adubação com calcário e fósforo (SG). Nas subparcelas foram aplicados quatro tratamentos de cobertura vegetal utilizando-se: Megathyrsus maximus B K Simon \& S W L Jacobs, capimtanzânia (m) com 38g de sementes por subparcela; Setaria sphacelata (Schum.) Staff \& C. E. Hubb ex Chip, capimkazungula (s) com 197g de sementes por subparcela; mistura de três gramíneas nativas Paspalum notatum Flügge, capimforquilha; P. regnellii Mez, macega-do-banhado; P. urvillei Steud (n) com 54; 12 e 19g de sementes por subparcela respectivamente e Exclusão (e). A densidade de semeadura utilizada foi função do respectivo valor cultural e a seleção das espécies em função do seu potencial para competir por luz com o capim-annoni-2.

Para avaliar a composição botânica e o tamanho do BSS coletou-se 10 subamostras de solo por subparcela, à profundidade de $10 \mathrm{~cm}$ e com $5 \mathrm{~cm}$ de diâmetro. $\mathrm{O}$ solo foi seco à sombra no período de nove dias, fragmentado e homogeneizado manualmente, preservando-se assim as sementes mais sensíveis quanto às injúrias mecânicas. Utilizou-se $30 \%$ da amostra homogeneizada, para a determinação da composição e do tamanho do BSS.

A identificação e quantificação das espécies do BSS foram realizadas em casa de vegetação através da germinação das sementes, presentes nas amostras. Utilizaram-se bandejas de alumínio com as dimensões de $12 \times 20 \times 5 \mathrm{~cm}$ revestidas com um filme de polietileno evitando desgaste precoce e possível efeito tóxico do cátion $\mathrm{Al}^{3}$ na germinação de sementes. A camada de solo nas bandejas foi de $3 \mathrm{~cm}$ de espessura. A este volume acrescentou-se $10 \%$ com vermiculita para melhor retenção da umidade nas bandejas e evitar a compactação e formação de crosta superficial do solo. Foram realizadas duas coletas, para o estudo do BSS, uma antes da instalação do experimento, em 26 de janeiro de 2005 e a segunda, após a aplicação dos tratamentos em 21 de maio de 2006. Foram realizados três ciclos de germinação com a finalidade de exaurir o BSS e remover a dormência das sementes viáveis remanescentes das duas coletas, com intervalos de suspensão de irrigação de 10 dias entre ciclos. À medida que o solo secava, fazia-se um revolvimento. As plântulas identificadas eram removidas, codificadas e herborizadas semanalmente. As plântulas de difícil identificação eram repicadas para vasos e identificadas em fases fenológicas posteriores. O turno de rega diário foi suficiente para manter o solo próximo à capacidade de campo.
$\mathrm{Na}$ análise dos dados, inicialmente utilizou-se da estatística descritiva como tamanho do BSS, médias de sementes.m ${ }^{-2}$, percentuais de participação das espécies na comunidade, coeficiente de variação e índice de Morisita, para identificar o padrão de distribuição espacial das espécies (Boccaneli e Lewis,1994; Medeiros e Steiner, 2002), calculada pela equação: I.M $=\sum \mathrm{n}(\mathrm{n}-1) / \mathrm{t}(\mathrm{t}-1) * \mathrm{~N}$. Onde $N$ é o número de amostras, $n$ é o número de sementes da espécie em cada amostra e $t$ é o total do número do indivíduo em todas as amostras. Se I.M $=1$ os indivíduos estão aleatoriamente dispersos, I.M $<0$ as espécies estão uniformemente distribuídas e I.M $>0$ as espécies estão agregadas.

Nas análises univariadas (variância e comparação das médias) adotou-se o aplicativo ASSISTAT versão 7.4 beta (Silva e Azevedo, 2006). Em função do elevado coeficiente de variação espacial, normalmente registrado em estudos de banco de sementes de solo (Bekker, 1998), utilizou-se também a análise multivariada com os aplicativos MULTIV 2.4.2 (Pillar, 2005a) e SYNCSA 2.2.4 (PILLAR, 2005b) buscando detectar padrões de variação. Na transformação utilizou-se a distância de corda. A congruência entre atributos de solo e BSS foi feita com a última avaliação, por conter os tratamentos na comunidade.

\section{RESULTADOS E DISCUSSÃO}

Nas duas avaliações do BSS, constatou-se a presença de 85 espécies, distribuídas entre 31 famílias. Destas espécies 68 surgiram na primeira coleta e 57 na segunda coleta do BSS. A maioria destas espécies possui ciclo de vida anual e pertencem às famílias Asteraceae e Poaceae, refletindo o padrão geral do Bioma Pampa.

O BSS compôs-se com $20 \%$ de espécies exóticas, evidenciando-se a potencialidade à invasão biológica, habilidades no estabelecimento e produção de descendentes, fato ocorrido com o capim-annoni-2. Na primeira avaliação a espécie Relbunium vile (Cham. \& Schltdl) K. Schum teve a maior quantidade de sementes, $2.529 . \mathrm{m}^{-2}$, ou $12,57 \%$ do BSS (20.110 sementes. ${ }^{-2}$ ) (Tabela 1). O capim-annoni-2 apresentou 963 sementes. m $^{-2}$, ou 4,79\% do BSS (Tabela 1). Na segunda avaliação, a espécie mais abundante foi Sisyrinchium micranthum Cav., com 3.896 sementes.m ${ }^{-2}$ ou $13,61 \%$ do BSS (28.617 sementes.m ${ }^{-2}$ ) (Tabela 1). O capimannoni-2 aparece com 2.040 sementes. $\mathrm{m}^{-2}$ ou $7,13 \%$ do BSS um acréscimo de 1.077 semente. $\mathrm{m}^{-2} \mathrm{em}$ relação ao primeiro levantamento. Solos em processo de distúrbios apresentam elevado número de sementes (Fenner, 1995). 
TABELA 1. Lista de espécies encontradas nos dois BSS com as respectivas espécies, famílias, espécies exóticas*, índice de Morisita, quantidade de sementes.m² ${ }^{-2}$, coeficiente de variação e percentual das espécies nos BSS.

\begin{tabular}{|c|c|c|c|c|c|c|c|c|c|}
\hline \multirow{2}{*}{ Espécies } & \multirow[t]{2}{*}{ FAMI } & \multicolumn{3}{|c|}{ BSS1 } & \multicolumn{5}{|c|}{ BSS2 } \\
\hline & & I.M. & S.m-2 & $\mathrm{CV}$ & $\mathrm{BSS} \%$ & I.M. & S.m-2 & $\mathrm{CV}$ & $\mathrm{BSS} \%$ \\
\hline Coniza bonariense & Aste & - & - & - & - & 4,6 & 1162 & 193,7 & 4,06 \\
\hline Aster squamatus & Aste & - & - & - & - & 3,7 & 432 & 167,3 & 1,51 \\
\hline Setaria sphacelata* & Poac & - & - & - & - & 5,2 & 248 & 210,1 & 0,87 \\
\hline Heliotropium indicum & Bora & - & - & - & - & 3,6 & 184 & 165,4 & 0,64 \\
\hline Nothoscordum sp & Lili & - & - & - & - & 3,4 & 184 & 158,5 & 0,64 \\
\hline Brisa subaristata & Poac & - & - & - & - & 6,0 & 85 & 228,4 & 0,30 \\
\hline Cyperus flavus & Cype & - & - & - & - & 8,6 & 64 & 281,5 & 0,22 \\
\hline Borreria verticillata & Rubi & - & - & - & - & 6,6 & 43 & 243,2 & 0,15 \\
\hline Apium leptophyllum & Apia & - & - & - & - & 24,0 & 28 & 489,9 & 0,10 \\
\hline Baccharis dracunculifolia & Aste & - & - & - & - & 13,3 & 21 & 358,7 & 0,07 \\
\hline Cuphea hysopifolia & Lyth & - & - & - & - & 13,3 & 21 & 358,7 & 0,07 \\
\hline Trifolium repens $*$ & Faba & - & - & - & - & 8,0 & 21 & 270,3 & 0,07 \\
\hline Amaranthus blitum * & Amar & - & - & - & - & 24,0 & 7 & 489,9 & 0,02 \\
\hline Anagalis arvensis * & Prim & - & - & - & - & 24,0 & 7 & 489,9 & 0,02 \\
\hline Megathyrsus maximus * & Poac & - & - & - & - & 24,0 & 7 & 489,9 & 0,02 \\
\hline Polygonum persicaria * & Poly & - & - & - & - & 24,0 & 7 & 489,9 & 0,02 \\
\hline Trifolium polymorphum & Faba & - & - & - & - & 24,0 & 7 & 489,9 & 0,02 \\
\hline Relbunium vile & Rubi & 2,5 & 2529 & 126,6 & 12,57 & 2,7 & 659 & 133,0 & 2,30 \\
\hline Piptochaetium montevidense & Poac & 1,6 & 2423 & 78,0 & 12,05 & 2,3 & 616 & 118,4 & 2,15 \\
\hline Sisyrinchium palmifolium & Irid & 1,7 & 1891 & 85,5 & 9,40 & 7,0 & 630 & 249,8 & 2,20 \\
\hline Gamochaeta spicata & Aste & 1,2 & 1842 & 45,7 & 9,16 & 1,7 & 2826 & 86,2 & 9,88 \\
\hline Anagallis minima $*$ & Prim & 2,1 & 1523 & 106,7 & 7,57 & 3,5 & 538 & 160,9 & 1,88 \\
\hline Eragrostis plana* & Poac & 1,3 & 963 & 53,7 & 4,79 & 1,4 & 2040 & 67,7 & 7,13 \\
\hline Heimia salicifolia & Lyth & 14,4 & 893 & 373,9 & 4,44 & - & - & - & - \\
\hline Oxalis corymbosa & Oxal & 1,8 & 751 & 93,5 & 3,73 & 1,7 & 326 & 88,3 & 1,14 \\
\hline Wahlenbergia linarioides & Camp & 2,0 & 595 & 100,4 & 2,96 & 1,9 & 1480 & 98,1 & 5,17 \\
\hline Triodanis biflora & Camp & 2,2 & 524 & 110,3 & 2,61 & 2,9 & 1310 & 139,4 & 4,58 \\
\hline Cerastium humifusum & Caro & 2,5 & 503 & 124,3 & 2,50 & 2,5 & 2876 & 125,0 & 10,05 \\
\hline Killinga brevifolia & Cype & 2,2 & 418 & 109,9 & 2,08 & - & - & - & - \\
\hline Hypoxis decumbens & Нyро & 1,7 & 390 & 86,8 & 1,94 & - & - & - & - \\
\hline Sisyrinchium micranthum & Irid & 2,2 & 390 & 112,5 & 1,94 & 3,2 & 3896 & 150,5 & 13,61 \\
\hline Cyperus piceus & Cype & 1,9 & 312 & 94,7 & 1,55 & - & - & - & - \\
\hline
\end{tabular}


N. R. FERREIRA et al.

\begin{tabular}{|c|c|c|c|c|c|c|c|c|c|}
\hline Callitriche deflexa & Call & 4,5 & 290 & 191,8 & 1,44 & 4,4 & 2805 & 187,4 & 9,80 \\
\hline Mollugo verticilleta & Aizo & 4,9 & 283 & 201,5 & 1,41 & 8,0 & 425 & 270,3 & 1,49 \\
\hline Verbena bonariense & Verb & 1,9 & 262 & 97,5 & 1,30 & 4,0 & 213 & 178,5 & 0,74 \\
\hline Scutellaria racemosa & Lami & 2,2 & 248 & 112,5 & 1,23 & - & - & - & - \\
\hline Coniza canadensis* & Aste & 2,3 & 248 & 117,9 & 1,23 & 4,2 & 255 & 182,3 & 0,89 \\
\hline Drymaria cordata $*$ & Cary & 2,2 & 234 & 111,0 & 1,16 & - & - & - & - \\
\hline Cyperus reflexus & Cype & 2,2 & 227 & 112,0 & 1,13 & - & - & - & - \\
\hline Veronica peregrina & Scro & 4,1 & 205 & 179,3 & 1,02 & 6,8 & 142 & 246,9 & 0,50 \\
\hline Hydrocotyle exigua & Apiac & 2,6 & 205 & 129,1 & 1,02 & 12,0 & 14 & 338,8 & 0,05 \\
\hline Plantago australis & Plan & 2,7 & 184 & 133,1 & 0,92 & 2,9 & 106 & 140,1 & 0,37 \\
\hline Soliva pterosperma & Aste & 3,6 & 156 & 163,8 & 0,77 & 3,5 & 425 & 161,3 & 1,49 \\
\hline Hybanthus parviflorus & Viol & 2,7 & 149 & 131,9 & 0,74 & 1,9 & 283 & 98,0 & 0,99 \\
\hline Dichondra sericea & Conv & 2,9 & 120 & 141,0 & 0,60 & 8,0 & 21 & 270,3 & 0,07 \\
\hline Eleusine tristachya & Poac & 2,6 & 113 & 130,2 & 0,56 & - & - & - & - \\
\hline Rhynchospora sp & Cype & 2,7 & 106 & 131,9 & 0,53 & 2,8 & 453 & 135,3 & 1,58 \\
\hline Cyperus sp & Cype & 6,7 & 92 & 243,3 & 0,46 & - & - & - & - \\
\hline Parietaria debilis* & Urti & 8,7 & 92 & 282,8 & 0,46 & 2,5 & 630 & 125,3 & 2,20 \\
\hline Paspalum plicatulum & Poac & 5,2 & 92 & 210,7 & 0,46 & 5,3 & 43 & 212,6 & 0,15 \\
\hline Cyperus luzulae & Cype & 4,3 & 71 & 186,1 & 0,35 & - & - & - & - \\
\hline Glandularia pulchella & Aste & 4,4 & 64 & 189,6 & 0,32 & 2,6 & 418 & 127,5 & 1,46 \\
\hline Paronchychia sp & Cary & 8,0 & 64 & 270,3 & 0,32 & 4,9 & 170 & 202,2 & 0,59 \\
\hline Erechtites valerianifolia & Aste & 3,7 & 57 & 169,4 & 0,28 & - & - & - & - \\
\hline Acanthospermum australe & Aste & 9,3 & 50 & 294,4 & 0,25 & 3,3 & 135 & 153,5 & 0,47 \\
\hline Oxalis sp & Oxal & 17,3 & 43 & 412,8 & 0,21 & - & - & - & - \\
\hline Richardia sp & Rubi & 4,0 & 43 & 176,9 & 0,21 & - & - & - & - \\
\hline Hydrocotyle leucocephala & Apiac & 24,0 & 35 & 489,9 & 0,18 & - & - & - & - \\
\hline Lepidium ruderale * & Bras & 4,8 & 35 & 199,1 & 0,18 & - & - & - & - \\
\hline Urtica circularis & Urti & 6,0 & 28 & 228,4 & 0,14 & - & - & - & - \\
\hline Diodia dasycephala & Rubi & 15,0 & 28 & 382,2 & 0,14 & 7,8 & 156 & 267,1 & 0,54 \\
\hline Eragrostis gloeodes & Poac & 6,0 & 28 & 228,4 & 0,14 & 24,0 & 7 & 489,9 & 0,02 \\
\hline Eryngium horridum & Apia & 8,0 & 21 & 270,3 & 0,11 & - & - & - & - \\
\hline Fimbristylis dichotoma & Cype & 13,3 & 21 & 358,7 & 0,11 & - & - & - & - \\
\hline Stemodia verticillata & Scro & 13,3 & 21 & 358,7 & 0,11 & 4,4 & 319 & 187,6 & 1,11 \\
\hline Paspalum urvillei & Poac & 13,3 & 21 & 358,7 & 0,11 & 5,9 & 220 & 225,4 & 0,77 \\
\hline Silene galica * & Cary & 8,0 & 21 & 270,3 & 0,11 & 2,6 & 170 & 128,5 & 0,59 \\
\hline Sida rhombifolia & Malv & 8,0 & 21 & 270,3 & 0,11 & 8,0 & 21 & 270,3 & 0,07 \\
\hline Sonchus oleraceus * & Aste & 8,0 & 21 & 270,3 & 0,11 & 8,0 & 21 & 270,3 & 0,07 \\
\hline
\end{tabular}




\begin{tabular}{|c|c|c|c|c|c|c|c|c|c|}
\hline Ambrosia elatior & Aste & 24,0 & 14 & 489,9 & 0,07 & - & - & - & - \\
\hline Baccharis articulata & Aste & 12,0 & 14 & 338,8 & 0,07 & - & - & - & - \\
\hline Cynodon dactylon * & Poac & 12,0 & 14 & 338,8 & 0,07 & - & - & - & - \\
\hline Solanum sisymbrifolium & Sola & 24,0 & 14 & 489,9 & 0,07 & - & - & - & - \\
\hline Vicia graminea & Faba & 24,0 & 14 & 489,9 & 0,07 & - & - & - & - \\
\hline Facelis apiculata & Aste & 12,0 & 14 & 338,8 & 0,07 & 3,2 & 893 & 150,5 & 3,12 \\
\hline Berroa gnaphahoides & Aste & 12,0 & 14 & 338,8 & 0,07 & 8,2 & 241 & 273,7 & 0,84 \\
\hline Erechtites hieracifolia & Aste & 24,0 & 7 & 489,9 & 0,04 & - & - & - & - \\
\hline Panicum hians & Poac & 24,0 & 7 & 489,9 & 0,04 & - & - & - & - \\
\hline Plantago tomentosa & Plan & 24,0 & 7 & 489,9 & 0,04 & - & - & - & - \\
\hline Senecio brasiliensis & Aste & 24,0 & 7 & 489,9 & 0,04 & - & - & - & - \\
\hline Setaria parviflora & Poac & 24,0 & 7 & 489,9 & 0,04 & - & - & - & - \\
\hline Chenopodium ambrosoides* & Chen & 24,0 & 7 & 489,9 & 0,04 & 3,7 & 269 & 166,5 & 0,94 \\
\hline Digitaria sanguinalis * & Poac & 24,0 & 7 & 489,9 & 0,04 & 12,0 & 14 & 338,8 & 0,05 \\
\hline Solanum americanum & Sola & 24,0 & 7 & 489,9 & 0,04 & 12,0 & 14 & 338,8 & 0,05 \\
\hline Sporobulus indicus & Poac & 24,0 & 7 & 489,9 & 0,04 & 24,0 & 7 & 489,9 & 0,02 \\
\hline Total & & & • & & & & 28.6 & & \\
\hline
\end{tabular}

Sendo as famílias: Aste (Asteraceae), Poac (Poaceae), Bora (Boraginaceae), Lili (Liliaceae), Cype (Cyperaceae), Rubi (Rubiaceae), Apia (Apiaceae), Lyth (Lythraceae), Faba (Fabaceae), Amar (Amaranthaceae), Prim (Primulaceae), Poly (Polygonaceae), Irid (Iridaceae), Oxal (Oxalidaceae), Camp (Campanulaceae, Caro (Carofilaceae), Hypo (Hypoxidaceae), Call (Callitrichaceae), Aizo (Aizoaceae), Verb (Verbenaceae), Lami (Lamiaceae), Cary (Caryophylaceae), Scro (Scrofulariaceae), Plan (Plantaginaceae), Viol (Violaceae), Conv (Convovulaceae), Urti (Urticaceae), Bras (Brassicaceae), Malv (Malvaceae), Sola (Solanaceae), Chen (Chenopodiaceae). FAMI = família; I.M = Índice de Morisita; $\mathrm{S} . \mathrm{m}^{-2}=$ quantidade de sementes por $\mathrm{m}^{2}$; $\mathrm{CV}=$ coeficiente de variação

Os distúrbios modificaram a constituição do BSS, onde 17 novas espécies surgiram, freqüência relativa de $8,8 \%$, duas delas introduzidas no ambiente (S. sphacelata e M. maximus). As demais gramíneas introduzidas, exceto P. urvillei que já existia em estado natural, não foram observadas no período, provavelmente em função de possíveis efeitos alopáticos, induzidos pelo capim-annoni-2, interferindo na germinação das espécies (Ferreira et al., 2006). Três espécies mantiveram a contribuição de sementes constante, 24 aumentaram, 14 diminuíram e 27 espécies desapareceram no segundo levantamento (Tabela1). O capim-annoni-2 confirmou sua condição de espécie oportunista com habilidade para ocupar espaços vazios provocados por distúrbios diversos, nas comunidades herbáceas (Reis, 1993; Reis e Coelho, 2000). As espécies que apareceram, suplantando o capim-annoni-2 em quantidade de sementes, são herbáceas, anuais, de porte baixo ou prostradas, do processo inicial de sucessão, mas com pouco potencial de concorrência com o capim-annoni-2 em função dos seus hábitos de crescimento.

As espécies introduzidas apareceram no segundo BSS, da seguinte forma: S. sphacelata, 248 sementes. $\mathrm{m}^{-2}$ ou $0,87 \%$ do BSS; P. urvillei, 220 sementes. $\mathrm{m}^{-2}$ ou $0,77 \%$ do BSS e M. maximus, com 7 sementes. $\mathrm{m}^{-2}$ ou $0,02 \%$ do BSS total (Tabela 1). Espera-se uma elevação destes montantes no BSS com a ressemeadura natural, na estabilização do sistema (Formoso, 1993), (Carámbula, 1997).

O índice de Morisita (IM) e coeficiente de variação $(\mathrm{CV})$ foram diretamente proporcionais e mostraram que a distribuição das espécies foi heterogênia no BSS. No primeiro BSS as espécies com menores I.M e CV foram Gamochaeta spicata e capim-annoni-2 com 1,2 e $45,7 \%$ e 1,3 e $53,7 \%$ respectivamente com dispersão aleatória. Resultados característicos de espécies invasoras, oportunistas e com grande amplitude ecológica. O mais alto índice de Morisita foi de 24 , com CV de 489,9\% representados por várias espécies com baixa freqüência no BSS, e em poucas parcelas com típica dispersão espacial agregada. No segundo BSS capim-annoni-2 manteve a mesma tendência do primeiro BSS, sendo a espécie com menor valor do I.M, com 1,4 e CV de $67,7 \%$. Os maiores valores também se igualaram aos do primeiro BSS. (Tabela 1).

As médias do BSS de capim-annoni-2, na interação dos 
tratamentos de solo com espécies introduzidas, apresentaram significância apenas na interação dentro da mistura de Paspalum spp. (Tabela 2). Os demais contrastes apenas indicaram tendência para tal. Os menores BSS ocorreram na interação subsolagem mais gradagem e adubação com as espécies introduzidas. Inferir-se que na subsolagem com posterior gradagem, muitas sementes de capim-annoni-2 atingiram camadas mais profundas do perfil do solo ficando no estoque do BSS, podendo vir a germinar em função de novos distúrbios. É reconhecido que quanto mais profundas estiverem as sementes de capim-annoni-2, menor será seu desaparecimento em relação às da superfície, e maior a sua preservação (Medeiros et al., 2005). A subsolagem com gradagem eliminou uma grande percentagem de plantas, levando a uma redução na freqüência e, menor produção de sementes. A menor perturbação do solo na subsolagem promoveu pouco prejuízo no ciclo de vida das plantas originais de capim-annoni-2 que estimuladas, revigoraram e produziram mais sementes. Finalmente o período entre os impactos da implantação do experimento e a segunda coleta do BSS (07-04-2005 a 21-05-2006) foi suficiente para que as sementes da superfície gerassem indivíduos adultos promovendo a ressemeadura natural com conseqüente incremento das diferenças.

TABELA 2. Médias do número de sementes de capim-annoni-2. $\mathrm{m}^{-2}$ no segundo BSS, na interação entre as subparcelas com os tratamentos de competição com espécies introduzidas mais a exclusão e os tratamentos de solo. Margem de rodovia Fazenda São Lucas, Rio Pardo RS / 2006.

\begin{tabular}{lccccc}
\hline \multirow{2}{*}{ Tratamento de solo } & \multicolumn{6}{c}{ Espécies } \\
\cline { 2 - 6 } & \multicolumn{1}{c}{ Mm } & Ex & Mp & Ss & Médias \\
\hline SG & 793,3 a A & 2436,7 a A & 793,3 b A & 1416,7 a A & 1360 a \\
S & 2436,7 a A & 3230,0 a A & 3400,0 a A & 1813,3 a A & 2720 a \\
\hline Médias & $1615,0 \mathrm{~A}$ & $2833,4 \mathrm{~A}$ & $2096,7 \mathrm{~A}$ & $1615,0 \mathrm{~A}$ & \\
\hline
\end{tabular}

Sendo, Mm, (subparcelas com Megathyrsus maximus), Ex, exclusão (subparcelas sem espécies introduzidas), Mp, (subparcelas com Mistura de Paspalum $\mathrm{ssp}$ ), Ss, (subparcelas com Setaria sphacelata), SG, (subparcelas com subsolagem mais gradagem) e S, (subparcelas com subsolagem). As letras minúsculas reportam às colunas e as maiúsculas às linhas, indicando que médias seguidas da mesma letra não diferem estatisticamente entre si pelo teste de Tukey a $5 \%$ de probabilidade. DMS para linha $=2994,0$ e para coluna $=2279,2 . \mathrm{CV}$ para tratamento de solo $=69,3 \%$ e para espécies $=60,5 \%$.

A comparação da variação entre o primeiro e o segundo BSS de capim-annoni-2 (BSS2-BSS1), mostrou significância nos contrastes da interação dos tratamentos de solo com $M$. maximus e mistura de Paspalum spp. Os demais contrastes, seguindo o mesmo padrão, tenderam para tal. Os dados sugerem que o sistema tratamento do solo com subsolagem mais gradagem e adubo teve forte influência no resultado final para reduzir a quantidade de sementes de capimannoni-2 do BSS (Tabela 3), em detrimento da subsolagem. Isto reforçando as conclusões das análises anteriores.

A análise multivariada do segundo BSS, apresentou a formação de quatro grupos nítidos (Figura 1), confirmada estatisticamente pelo teste de autoreamostragem (bootstrap).

TABELA 3. Comparação da variação do BSS do capim-annoni-2 (BSS 2-BSS 1), em sementes.m² na interação espécies introduzidas versus tratamento de solo. $\mathrm{Mm}=$ Megathyrsus maximus,. Margem de rodovia, Fazenda São Lucas, Rio Pardo, RS 2006.

\begin{tabular}{lccccc}
\hline \multirow{2}{*}{ Tratamento Solo } & \multicolumn{5}{c}{ Espécies } \\
\cline { 2 - 6 } & \multicolumn{1}{c}{ Mm } & Ex & Mp & Ss & Média \\
\hline SG & $-847,8$ b A & 1474,6 a A & $-564,8$ b A & 681,0 a A & 185,8 a \\
S & 1870,8 a A & 2381,2 a A & 2777,5 a A & 851,3 a A & 1970,2 a \\
\hline Média & $511,5 \mathrm{~A}$ & 1927,9 A & $1106,4 \mathrm{~A}$ & $766,2 \mathrm{~A}$ & \\
\hline
\end{tabular}

Ex $=$ Exclusão, $\mathrm{Mp}=$ Mistura de Paspalum spp., $\mathrm{Ss}=$ Setaria sphacelata Nas colunas, letras minúsculas, nas linhas, letras maiúsculas. DMS linhas $=2979,9$, DMS coluna $=2158,8 . \mathrm{CV}$ do tratamento de solo $=19,8 \%$ e CV das espécies $=20,2$. As médias seguidas da mesma letra não diferem estatisticamente entre si pelo teste de Tukey no nível de $5 \%$ de probabilidade. 


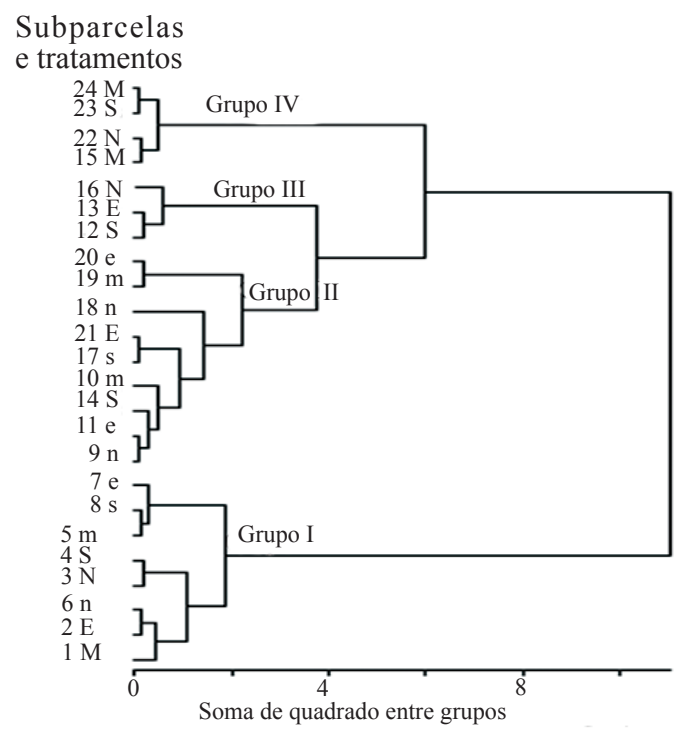

FIGURA 1. Dendrograma da análise de agrupamento do segundo BSS, obtido pela soma dos quadrados (variância mínima), formando quatro grupos nítidos a partir das subparcelas, com seus respectivos tratamentos. Onde, os números de 1 a 24 são as unidades amostrais, as letras maiúsculas significam os tratamentos de subsolagem, gradeamento e adubação. As letras minúsculas significam o tratamento subsolagem. As letras " $\mathrm{m} "=$ Megathyrsus maximus, "e" = exclusão, "n" = Paspalum spp., "s" = Setaria sphacelata. Margem de rodovia, Fazenda São Lucas, Rio Pardo / RS, 2006.

As subparcelas reunidas em grupos possuem um grau de similaridade suficiente para agregá-las por características comuns (Valentin, 2000). Os efeitos da aplicação dos tratamentos de preparo do solo tiveram forte influência na formação dos grupos. O grupo II, composto de nove subparcelas teve sete delas tratadas por subsolagem. Na subsolagem registraram-se os maiores tamanhos do BSS de capim-annoni-2 com $52,4 \%$ do total. Este fato confirma as inferências das análises univariadas quanto à observação de que maiores números de sementes acumularam-se nas camadas mais profundas do solo, as quais, em função de novos distúrbios poderiam vir à superfície e oportunizar a germinação das sementes viáveis. No grupo IV, as quatro subparcelas que o compôs, receberam o tratamento de subsolagem mais gradagem e adubação, evidenciando este fator como aglutinador do grupo. O grupo I, formado pelas unidades amostrais de um a oito teve como fator agregante às características de relevo (em platô), com melhores condições físico-químicas do solo e melhor retenção de água, suplantando os efeitos dos tratamentos. Tais atributos de solo promoveram uma maior diversidade de espécies. Das 57 espécies do BSS2, 52 aparecem no grupo I, que possui $67,67 \%$ da quantidade total de sementes da área de estudo, sendo que apenas $3,62 \%$ das sementes do grupo I são de capim-annoni-2. As outras são mais afetadas por processos erosivos. O grupo III, constituído pelas subparcelas 12, 13 e 16, apresentou características de baixo estabelecimento das espécies introduzidas. Este grupo localizou-se no setor mais erodido do estudo refletindo o baixo número de sementes encontrada, $6,24 \%$ do total. Deste percentual 58,33\% representado pelo capim-annoni-2, mostrando a rusticidade e adaptabilidade desta espécie em ocupar áreas degradadas.

A condensação das informações de semelhanças ecológicas em cada eixo é responsável pela ordenação das amostras (Valentin, 2000). A ordenação dos dados apresentou o diagrama de dispersão com localização das subparcelas e espécies em correlações acima de 0,6 com os eixos I e II (Figura 2). O eixo I explica $32,3 \%$ da variância dos dados e o eixo II, 21,6\%. Portanto, o diagrama representa 53,9\% da variação total das unidades amostrais. A ordenação confirma o observado no agrupamento (quatro grupos nítidos). No eixo I correlacionaram-se positivamente: Hybanthus parviflora $(0,65)$ e Eragrostis plana $(0,64)$. Associadas negativamente têm-se: Callitriche deflexa $(-0,82)$; Sisyrinchium micrantum $(-0,80)$ e Mollugo verticillata $(-0,65)$. No eixo II: Eragrostis plana $(0,73)$ e negativamente: Gamochaeta spicata $(-0,74)$ e Wahlenbergia linariodes $(-0,68)$. O grupo 2 é onde se encontrará maior BSS do capim-annoni-2, sendo representado pelas unidades amostrais $(9,10,11,14,17,18,19,20,21)$, de acordo com o dendrograma de agrupamento. Os eixos de ordenação apresentaram tendência à significância a partir de teste de autoreamostragem (bootstrap) com o limiar de 0,1 .

Avaliou-se ainda o nível de congruência ou correlação matricial entre o segundo BSS e os atributos de solo (Figura 3). O nível de correlação varia no intervalo de 0 a 1 e indica quais as variáveis que estão relacionadas com a composição do BSS. As correlações de variáveis cumulativas situaramse entre 0,58 com o fator zinco $(\mathrm{Zn})$ a 0,46 com todas as variáveis. Os atributos mais significativos foram: zinco, saturação do alumínio da CTC, CTC e fósforo. Este resultado demonstra a nítida relação entre BSS e alguns atributos de solo, refletindo nos impactos nas margens viárias com surgimento de mosaicos de níveis fertilidade e conseqüente interferência no BSS. 


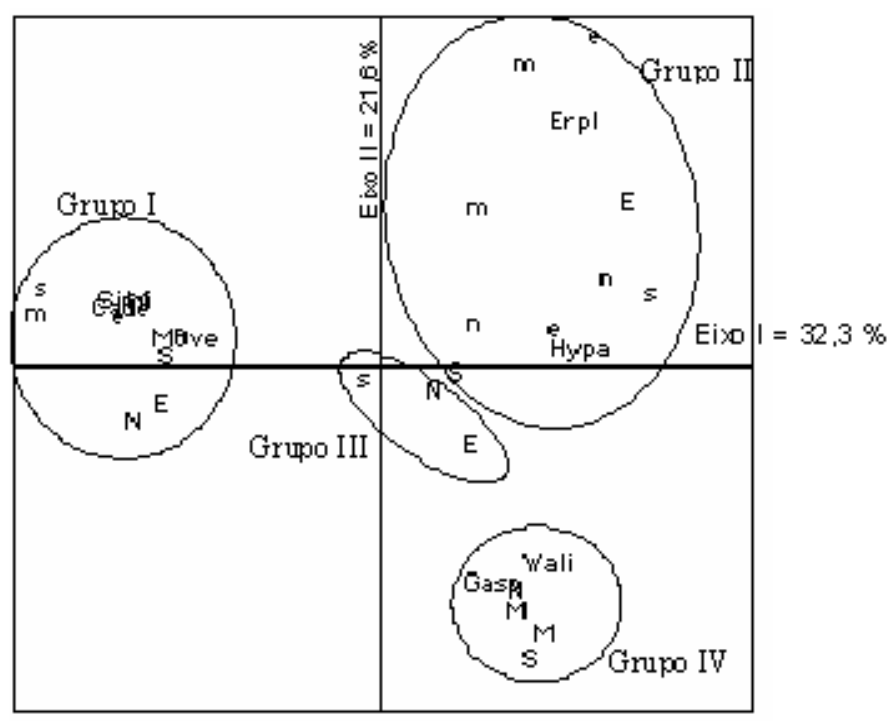

FIGURA 2. Diagrama de dispersão com correlações maiores do que 0,6 , obtido por análise das coordenadas principais (PCOA), com base em distância de corda e dados do segundo BSS, nos eixos I e II, explicando 53,9\% das variações no BSS, e os quatro grupos formados: Hypa (Hybanthus parviflora), Erpl (Eragrostis plana), Cade (Callitriche deflexa), Simi (Sisyrinchium micrantum), Move (Mollugo verticillata), Gasp (Gamochaeta spicata) e Wali (Wahlenbergia linariodes). Margem de rodovia, Fazenda São Lucas, Rio Pardo / RS, 2006.

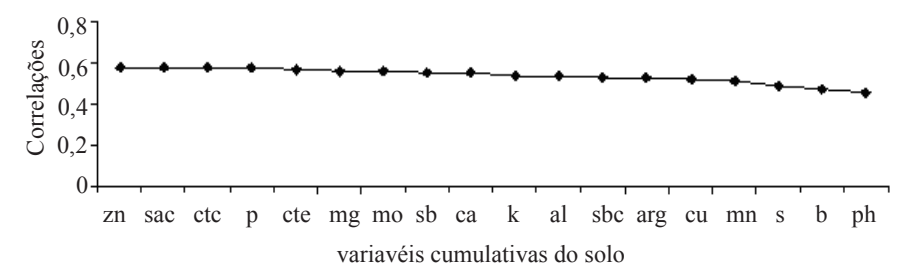

FIGURA 3. Perfil de congruência máxima entre a va-riação do levantamento do segundo BSS e os atributos de solo. Sendo cumulativamente da esquerda para a direita: zn (zinco); sac (saturação do alumínio da ctc efetiva); ctc (capacidade de troca de cátions); $p$ (fósforo); cte (capacidade de troca de cátions efetiva); mg (magnésio); mo (molibdênio); sb (soma de bases); ca (cálcio); k (potásio); al (alumínio); sbc (soma de base da ctc); arg (argila); cu (cobre); mn (manganês); s (enxofre); b (boro); ph (pH).

\section{CONCLUSÕES}

O acostamento viário apresenta $20 \%$ do BSS composto por espécies exóticas, nas quais o capim-annoni-2 se sobressai, sendo sua abundância estimulada pelos distúrbios no ambiente.

As espécies que mais inibem o BSS do capim-annoni-2 são Setaria sphacelata e Megathyrsus maximus, principalmente quando associada à subsolagem acompanhada de gradagem e adubação, podendo ser indicadas para controlar esta invasora em acostamento de rodovias.

O distúrbio provocado pelo preparo do solo com subsolagem seguido de gradagem e adubação promove maior redução do BSS de capim-annoni-2 do que subsolagem isolada.

A presença de sementes de um expressivo número de famílias e espécies botânicas no solo indica alto potencial de regeneração da vegetação nativa em acostamentos de rodovias dominadas por capim-annoni-2.

Os atributos de solo mais associados à composição do BSS são: zinco, saturação do alumínio da CTC, CTC e fósforo.

O BSS de capim-annoni-2 não apresenta padrão definido em função de sua grande amplitude de adaptabilidade ambiental, característica das espécies invasoras.

\section{AGRADECIMENTOS}

Os autores agradecem à Fazenda São Lucas $\left(\mathrm{Sr}^{\mathrm{a}} \mathrm{Nilza}\right.$ Barnetch e Sr. Alexandre Zarpellon), ao Sindicato Rural de Rio Pardo - RS, a CAPES e ao PROPESC/UFRGS pelo apoio logístico e ou financeiro.

\section{REFERÊNCIAS}

BEKKER, R. M. The ecology of soil seed bancks in grassland ecosystems. Amsterdam: VAN DENDEREN BV., 1998.193p.

BOCCANELLI, S. I.; LEWIS, J. P. The seed bank of an old pampean praire and relation with the standing vegetation. Pesquisa Agropecuária Brasileira, Brasília, v.29, n.12, p.1833-1840, 1994.

BOLDRINI, I. I.; KAMPF,A. N. Composição botânica dos campos naturais das estações experimentais da secretaria da agricultura RS - relação ilustrada de gramíneas. Anuário Técnico do Instituto de Pesquisa Zootécnica "Francisco Osório", Porto Alegre, v.4, p.233-266, 1977. 
CARÁMBULA, M. Pasturas naturales mejoradas. Montevidéu: HEMISFERIO SUR, 1997. 524p.

CAVERS, P. B. Seed banks: memory in soil. Canadian Journal of Soil Science, Ottawa, v. 75, n.1 p.11-13, 1995.

CONSTANZA, R. Ecological economics: the science and management of sustentability. New York:Columbia University Press, 1991.

FENNER, M. Ecology of seed banks. In: KIGEL, J.; GALILI, G. (Ed.). Seed development and germination. New York: Marcel Dekker, 1995. p. 507-528.

FERREIRA, N. R.; MEDEIROS, R. B.; SOARES, G. L. G. Avaliação alelopática do Capim-Annoni-2 sobre a germinação de sementes de gramíneas perenes. In: REUNIÃO DO GRUPO TÉCNICO EM FORRAGEIRAS DO CONE SUL GRUPO CAMPOS, 21., 2006, Pelotas. Anais... Pelotas: EMBRAPA, 2006. 1CD-Rom. cod. 6-08.

FORMAN, R. T. T.; HEANUE, K. Road ecology - science and solutions. Whashington: Island Press, 2002. 424p.

FORMOSO, F. Lotus corniculatus: Performance forrajera y caracteristicas agronómicas asociadas. INIA, Montevideo. Série Técnica n.2, p. 1-20, 1993.

KISSMANN, K. G. Plantas Infestantes e Nocivas. São Paulo: Basf Brasileira, 1991. Tomo I. 603p.

MEDEIROS, R. B.; FOCHT, T.; FERREIRA, N. R.; BRACK, S. C. F. 2004. Longevidade de Sementes de Eragrostis plana Nees em um Solo de Campo Natural. In: Reunión del Grupo Técnico Regional del Cono Sur en Mejoramiento y Utilización de los Recursos Forrajeros del Área Tropical y Subtropical - Grupo Campos, XX. 28 a 30 de Setiembre, 2004. Memorias...Universidad de la Republica: Salto, Uruguay. p. 213-214.

MEDEIROS, R. B; FOCHT, T.; FERREIRA, N. R.; FREITAS, M. R.; MENEGON, L. L. Longevidade de sementes de capim-annoni-2 (Eragrostis plana Nees) num solo de campo natural da depressão central do RS. In: CONGRESSO BRASILEIRO DE SEMENTE, 14., 2005, Foz do Iguaçu. Anais... Brasília: ABRATES, 2005. Publicado em Informativo ABRATES, Brasília, v. 15, p. 125-125.

MEDEIROS, R. B.; STEINER, J. J. Influência de sistemas de rotação de sementes de gramíneas forrageiras temperadas na composição do banco de sementes invasoras no solo. Revista brasileira de sementes, Brasília, v. 24, n.1, p. 118128, 2002.

ODUM, E. P. Ecologia. Rio de Janeiro: Guanabara, 1988. $434 \mathrm{p}$.

PICKETT, S. T. A.; WHITE, P. S. The ecology of natural disturbance and patch dynamics. San Diego: Academic Press, 1985. 455p.

PILLAR, V. D. P. MULTIV: aplicativo para análise multivariada e teste de hipóteses - versão 2.4.2, Porto Alegre: Departamento de Ecologia da UFRGS, 2005 a.

PILLAR, V. D. P. SYNCSA: software integrado para análise multivariada de comunidades baseada em caracteres, dados de ambiente, avaliação e testes de hipóteses - versão 2.2.4. Porto Alegre: Departamento de Ecologia da UFRGS, 2005 b.

PRIMAVESI, A. Manejo ecológico do solo: agricultura em regiões tropicais. São Paulo: Nobel,1979. 549p.

REIS, J. C. L. Capim-Annoni-2: origem, morfologia, características, disseminação. In: REUNIÃO REGIONAL DE AVALIAÇÃO DE PESQUISA COM ANNONI-2, 1993, Bagé. Anais... Bagé: EMBRAPA-CPPSUL, 1993. d.7, p.519.

REIS, J. C. L.; COELHO, R. W. Controle do CapimAnnoni-2 em campos naturais e pastagens. Pelotas. Embrapa Clima Temperado, 2000. c.t,22, p.21, 2000.

SILVA, F. A. S.; AZEVEDO, C. A. V. A new version of the assistat - statistical assistance software. In: WORD CONGRESS ON COMPUTERS IN AGRICULTURE, 4., Orlando, FL, USA. Anais... Orlando: American Society of Agricultural Engineers, 2006. p. 393-396.

STRECK, E. V.; KÄMPF, N.; DALMOLIN, R. S. D.; KLAMT, E.; NASCIMENTO, P. C.; SCHEIDER,P. Solos do Rio Grande do Sul. Porto Alegre: UFRGS, 2002. 107p.

VALENTIN, J. L. Ecologia numérica: uma introdução à análise multivariada de dados ecológicos. Rio de Janeiro: Interciência, 2000. 117p. 